\title{
Nonlinear Wave Mixing in Semiconductor Nanoantennas and Metasurfaces
}

\author{
Dragomir N. Neshev \\ Nonlinear Physics Centre, Research School of Physics and Engineering, The Australian National University, Canberra, Australia \\ Dragomir.Neshev@anu.edu.au
}

\begin{abstract}
We demonstrate how ultra-small dielectric nanoantennas ordered in a metasurface can enable enhanced light matter interaction for efficient nonlinear wave-mixing and in particular, to enhance sum-frequency generation and spontaneous parametric down conversion. OCIS codes: (160.3918) Metamaterials; (190.4975) Parametric processes
\end{abstract}

\section{Introduction}

Frequency conversion of light is one of the most fundamental processes of nonlinear optics and can be achieved by interaction of light beams in nonlinear crystals. However, such processes are considered unrealistic in small nanocrystals due to the negligible efficiency. Nevertheless, researchers have been actively looking for ways of increasing the efficiency of nonlinear frequency conversion at the nanoscale [1]. Plasmonic metasurfaces were first considered for nonlinear frequency conversion. However, due to the limited volume of the plasmonic modes, the overall nonlinear conversion efficiency is small. Metasurfaces composed of dielectric nanostructures have recently shown better performance due to the excitation of Mie resonances and the larger interaction volume [2]. A record high frequency conversion enhancement has been recently demonstrated in AlGaAs nanodisks (having quadratic nonlinear susceptibility), which can enable second harmonic generation (SHG) efficiency of 10 ${ }^{-4}$ [3-5]. These record-higher efficiencies open a wide range of possible applications, including nonlinear microscopy and holography. In this work, we review the recent progress of nonlinear frequency mixing in all-dielectric metasurfaces and explain the underlying physics behind the enhancement of the nonlinear processes in high-refractive index nanocrystals, including AlGaAs. We further show how the resonant enhancement of the spontaneous parametric down conversion (SPDC) process in the AlGaAs nano-resonator leads to non-classical photon polarization-correlations.

\section{Second harmonic generation in AlGaAs nanocrystals}

The key to achieve high conversion efficiency of the nonlinear processes, such a SHG is for the dielectric nanoantennas to satisfy several requirements: $(i)$ having high refractive index for high-quality factor resonances at all participating wavelengths; (ii) lacking inversion symmetry of the material crystalline lattice - to enable second order nonlinear effect with nonlinear susceptibility $\chi^{(2)}$; and (iii) engineer the directionality of the antenna emission for the light to efficiently collected by the observer. One material matching these criteria is the GaAs and its compounds, e.g. AlGaAs. Recent works [3, 4, 5] have shown state-of-the-art fabrication of AlGaAs nanodisk antennas on a low index substrate. With this unique fabrication of AlGaAs nanostructures, we could fully explore the efficient nonlinear frequency conversion in AlGaAs nanocrystals and their radiation direction. However the the observed radiation pattern was shown to represent a doughnut shape, thus not emitting at normal direction [5]. This questioned the possible implementation of such nanoantennas for practical applications, such us nonlinear imaging. To engineer the radiation pattern from a single antenna and enable radiation at normal direction, we used the technique of leaky wave antennas, to redirect the in-plane radiation towards the normal direction with an engineered phase. This approach is shown in Fig.la and not only allows for emitting SHG at normal direction, but also to engineer the specific radiation pattern to create holographic images of different shapes.

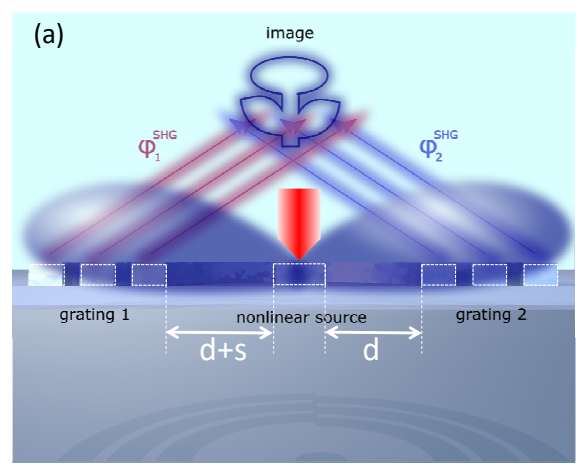

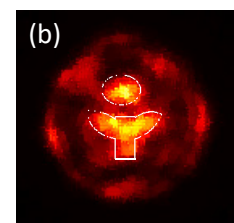
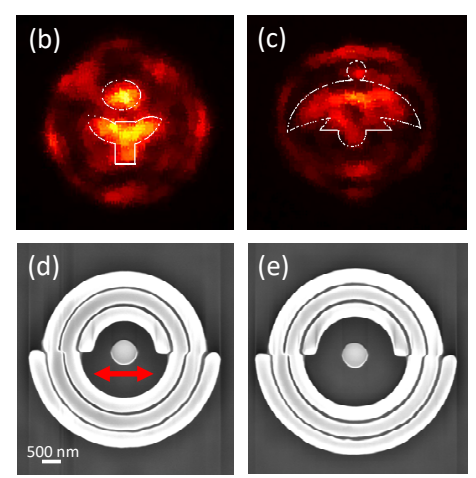

Figure 1: (a) Engineering of the radiation pattern of second harmonic generation by holographic gratings. (b-e) SHG holography in the far field radiation pattern (back-focal-plane), which creates a virtual image resembling a flower (b) and a bird (c). (d,e) SEM images of the corresponding nanostructures having: (c) $\mathrm{d}=500 \mathrm{~nm}$, and (e) $\mathrm{d}=750 \mathrm{~nm}$. 
The measured holographic radiation patterns and the corresponding scanning electron micrograph (SEM) images of the designed nonlinear antennas are shown in Fig.1b-e. These results demonstrate the power of our concept to enable normal radiation emission from AlGaAs nanoantennas and to allow for nonlinear holography with single nanoantennas, having possible applications in nonlinear microscopy and security holograms.

\section{Beyond harmonic generation: sum and difference frequency mixing}

The measured SHG from individual AlGaAs nanoantennas open new opportunities to study mixing of different colors in nonlinear nanocrystals in the regime, beyond harmonic generation. As an example, we have investigated the process of sum frequency generation (SFG) from an [100] AlGaAs-on-oxide monolithic nano-resonator [4], as shown in Fig. 2a. We use two wavelengths from a broad-spectrum femtosecond laser, namely a signal beam at $1520 \mathrm{~nm}$ and an idler beam at $1560 \mathrm{~nm}$ (Fig. 2b). We combine these two beams and focus them onto a single $400 \mathrm{~nm}$-high and $420 \mathrm{~nm}$-wide AlGaAs nanodisk resonator using a $0.7 \mathrm{NA}$ objective. The polarization in each arm can be controlled independently. We vary the optical delay between both pulses and measure the spectrum of the newly generated light, in reflection. We observe three spectral traces (Fig. 2c): two, which are independent on the time delay, at $760 \mathrm{~nm}$ and $780 \mathrm{~nm}$, correspond to SHG by the signal and idler, respectively. The third trace at $770 \mathrm{~nm}$ is much stronger and appears only at the complete overlap between the two pulses and corresponds to sumfrequency generation (SFG) and can be observed for different input polarizations of the signal and idler pulses, as shown in Fig.2d. The SFG peak appears stronger than the SHG peaks due to directional distribution of the energy to the detector. These results show that mixing of arbitrary colors is possible in nonlinear dielectric metasurfaces, thus opening novel opportunities for nonlinear imaging and night vision. The observed SFG efficiency further brings confidence for the possibility to generate single photons at the nanoscale using the inverted SPDC process.
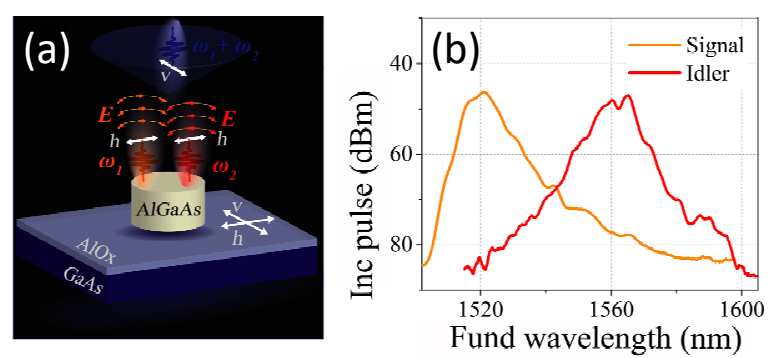
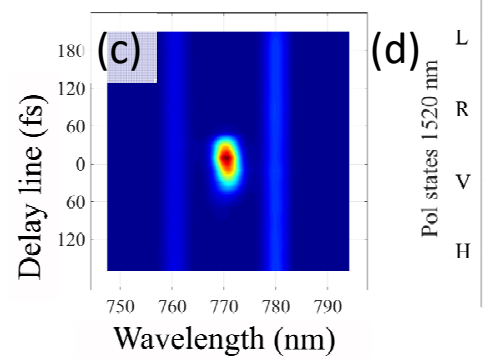

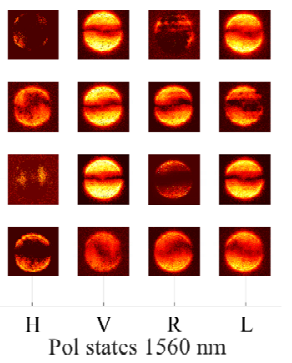

Figure 2: (a) Schematic the experimental arrangement for SFG wave-mixing. (b) Signal (orange line) and idler spectrum (red line) filtered from the fs laser. (d) Time dependent spectrum of the nonlinear wave mixing in the AlGaAs nano-disk as a function of the time delay between the signal and idler pulses. The SFG only happens when the two pulses overlap, while the spectral features at 760 and $780 \sim \mathrm{nm}$ correspond to SHG from the individual signal and idler pulses. (d) Measured reflected SFG directionality diagrams for different polarizations an NA=0.7.

\section{Conclusions}

The of mixing of colors with high efficiency in nonlinear dielectric nanoantennas and metasurfaces offers unique opportunities for novel types of applications. For example, applications in nonlinear microscopy can be envisaged. Furthermore, ultra-secure nonlinear holograms can be designed based on SHG nanocrystal to help against fraud in documents or banknotes. In general, the observed THG, SHG, SFG and SPDC nonlinear process can be extended towards DFG and parametric amplification. One can even imagine, nonlinear metasurfaces placed on top of eyeglasses to convert infra-red to visible light for night vision. There are endless opportunities ahead, however a lot of research still needs to be done to deeply understand the physics of nonlinear frequency conversion at the nanoscale.

\section{Acknowledgements}

We acknowledge the contribution of G. Marino, L. Ghirardini A. Solntsev, L. Xu, R. Camacho-Morales, V. Gili, I. Favero, D. Rocco, L. Carletti, A. Poddubny, M. Rahmani, D. Smirnova, H. Chen, A. Locatelli, M. Finazzi, M. Celebrano, H. Tan, F. Karouta, A. Zayats, C. Jagadish, C De Angelis, G Leo, Y. Kivshar, and A. Sukhorukov.

\section{References}

1. M. Kauranen and A. V. Zayats, "Nonlinear plasmonics," Nat. Photon. 6, 737-748 (2012).

2. M. R. Shcherbakov, D. N. Neshev, B. Hopkins, A. S. Shorokhov, I. Staude, E. V. Melik-Gaykazyan, M. Decker, A. A. Ezhov, A. E. Miroshnichenko, I. Brener, A. A. Fedyanin, and Y. S. Kivshar, Nano Lett. 14, 6488-6492 (2014).

3. V. F. Gili, L. Carletti, A. Locatelli, D. Rocco, M. Finazzi, L. Ghirardini, I. Favero, C. Gomez, A. Lemaître, M. Celebrano, C. De Angelis, and G. Leo, Optics Express 24, 15965-15971 (2016).

4. S. Liu, M. B. Sinclair, S. Saravi, G. A. Keeler, Y. Yang, J. Reno, G. M. Peake, F. Setzpfandt, I. Staude, T. Pertsch, and I. Brener, Nano Lett. 16, 5426-5432 (2016).

5. R. Camacho-Morales, M. Rahmani, S. Kruk, L. Wang, L. Xu, D. A. Smirnova, A. S. Solntsev, A. Miroshnichenko, H. H. Tan, F. Karouta, S. Naureen, K. Vora, L. Carletti, C. De Angelis, C. Jagadish, Y. S. Kivshar, and D. N. Neshev, Nano Lett. 16, $7191-7197$ (2016). 